\title{
Focus on nutrition
}

\author{
Pierre Singer ${ }^{1 *}$, Giuseppe Citerio ${ }^{2}$ and Gordon Doig ${ }^{3}$
}

๑ 2016 Springer-Verlag Berlin Heidelberg and ESICM

\section{Introduction}

"The truth on nutrition in the ICU" [1], published early last year, identified a number of basic truths about nutrition support that were supported by evidence. Many of these truths have been confirmed in the most recent publications during 2015 related to nutrition in the intensive care.

The strongest evidence identified by the early 2015 review paper was related to early enteral nutrition starting [1] and this concept was confirmed by Reigner et al. in a post-marginal structural model study on the impact of early nutrition on outcome of ventilated patients in shock [2], a topic in active debate. In 3032 patients in shock, early enteral or parenteral nutrition commenced within $48 \mathrm{~h}$ after intubation was associated with reduced mortality. Interestingly, early calories intake administered after initial hemodynamic stabilization, when high doses of catecholamines may still be administered, was associated with reduced mortality. Early enteral feeding was, however, associated with increased risk of ventilator-associated pneumonia, but this did not translate to increased mortality.

The fear of acute mesenteric ischemia in the ICU remains present especially when feeding patients after initial hemodynamic stabilization of shock. Leone et al. addressed this issue in a 6-year retrospective survey in 46 ICUs involving 780 patients suffering from acute mesenteric ischemia [3]. Whilst $58 \%$ of the patients identified with acute mesenteric ischemia died, surprisingly, the survivors were found to have been receiving more enteral feeding at time of diagnosis, confirming the previous findings of Reigner et al. [2].

Over the past year, the Society of Critical Care Medicine (SCCM) and the American Society for Parenteral

\footnotetext{
*Correspondence: psinger@clalit.org.il

${ }^{1}$ Department of Critical Care and Institute for Nutrition Research, Rabin Medical Center, Beilinson Hospital, Sackler School of Medicine, Tel Aviv University, Tel Aviv, Israel

Full author information is available at the end of the article
}

and Enteral Nutrition (ASPEN) have released new guidelines on nutrition in the ICU [4], and the expert consensus process resulted in some contradictory messages. Perhaps the most controversial expert recommendation is that patients with a normal BMI who cannot receive enteral nutrition (EN) or oral intake can be starved for 7 days. The recommendation to withhold parenteral nutrition over the first 7 days following ICU admission if early enteral nutrition is not feasible contradicts evidence from large-scale randomized controlled trials (RCTs) that demonstrate that early parenteral nutrition (PN) preserves muscle mass, shortens the duration of mechanical ventilation, and improves quality of life postICU discharge [1]. Indeed, after the publications of the CALORIES [5] study showing no harm related to PN in ICU patients, unfounded biases regarding the detrimental effect of early PN must be reconsidered together with the amount of calories administered [1]. Elke et al. [6], in a review of the literature, compared PN to EN when the calories administered were similar and could not find any difference in terms of morbidity and mortality.

Anabolic resistance has been suggested [1] as a main obstacle to improving nitrogen balance. Protein has to be administered in large amounts [7] and exercise has been suggested to be integrated into the nutritional therapy. The energy cost of early exercise in critically ill patients has been studied by Hickmann et al. over the past year [8]. Three out of 60 patients suffered physical exhaustion and had to stop the physical activity (cycle ergometry). Energy expenditure was significantly higher during exercise in ICU patients compared to volunteers and this finding should be important in planning calories requirements. The best planning may be achieved using indirect calorimetry $[4,9]$.

The pharmaconutrition approach is also a matter of debate. Intravenously administered glutamine in trauma patients [10] did not show any clinical advantages, but $60 \%$ of the patients presented low glutamine levels before randomization and $48 \%$ maintained low levels 
after 6 days of treatment. Those with low levels at day 6 had significantly more infection, longer ICU, and hospital stay. The same trend was found by Kagan et al. [11] after preemptively administrating enteral feeding enriched in omega-3 fatty acids and gamma-linolenic acid in trauma patients. Again no difference was found between the study and the control group but levels of membrane omega-3 index failed to reach therapeutic levels even after 7 days of therapy. The optimal amount of glutamine or EPA supplementation remains unknown and a definitive conclusion regarding pharmaconutrition cannot be reached at this time. Similarly, a meta-analysis of studies comparing ICU patients receiving supplemental enteral omega-3 fatty acids [12] did not find any advantage in favor of omega-3 fatty acids but this analysis included studies administrating omega-3 fatty acids continuously or by bolus, at difference doses, and to different populations, thereby leaving the conclusions questionable.

The relationship between glycemic control and patient outcomes has also been addressed in recent publications. In a well-conducted clinical trial enrolling 2684 patients expected to require at least 3 days of ICU care, computerized tight glycemic control $(4.4-6.1 \mathrm{mmol} / \mathrm{L})$ was compared to conventional $(<10 \mathrm{mmol} / \mathrm{L})$ control [13]. Although the authors report no significant difference in the primary outcome, i.e., day 90 mortality, the $95 \%$ confidence interval around the risk difference $(32.2$ vs $34.1 \%, 95 \% \mathrm{CI}-5$ to $2 \%$ ) includes the treatment effects reported in previous trials addressing this issue [1]. Unfortunately, computerized tight glycemic control was also associated with a significant increase in moderate and severe hypoglycemic events.

In addition to new information generated from this most recent clinical trial, the NICE-SUGAR investigators reported long-term follow-up (2 year) on an a priori identified subgroup of traumatic brain injury (TBI) patients [14]. Interestingly, although day 90 mortality was not significantly different between groups $(P=0.20)$, it appeared to be $4.2 \%$ lower in patients randomized to receive intensive $(4.5-6.0 \mathrm{mmol} / \mathrm{L})$ insulin control. Whilst a formal test of heterogeneity was not presented in this publication, when the apparent $4.2 \%$ reduction in mortality in TBI patients was compared to the significant $3 \%$ excess day 90 mortality reported in NICE-SUGAR for the 5631 patients enrolled without TBI, there is a suggestion that TBI patients could respond differently. Although 2-year mortality $(P=0.60)$ and 2-year Glasgow Outcome Score $(P=0.43)$ did not differ between groups, larger studies may be required to rule out benefits. TBI patients randomized to intensive glycemic control did experience significantly more moderate and severe hyopoglycemic events, so we recommend that sensitive assessments of neurocognitive function should be undertaken during future trials in order to detect possible long-term sequelae of hypoglycemic events that may be important to patients.

Also of interest over the past year, a well-conducted systematic review of clinical trials assessing stress ulcer prophylaxis was reported [15]. These authors identified 20 clinical trials enrolling 1971 patients. This comprehensive review reported that neither proton pump inhibitors nor $\mathrm{H}_{2}$ receptor antagonists reduced mortality; however, compared to placebo, $\mathrm{H}_{2}$ receptor antagonists were demonstrated to significantly reduce gastrointestinal bleeding.

In summary, the past year has resulted in some interesting publications in the field, mostly confirming knowledge available at the start of the year but also providing important insights into the provision of EN to patients with recently stabilized shock. The answers regarding when to start, which route to choose, how many calories and how much protein to prescribe, and how to progress through ICU hospitalization need more studies integrating all these parameters to be able to give a definitive answer.

\section{Author details \\ ${ }^{1}$ Department of Critical Care and Institute for Nutrition Research, Rabin Medical Center, Beilinson Hospital, Sackler School of Medicine, Tel Aviv University, Tel Aviv, Israel. ${ }^{2}$ School of Medicine and Surgery, University of Milan-Bicocca, Monza, Italy. ${ }^{3}$ Intensive Care Unit, Royal North Shore Hospital, St Leonards, NSW 2065, Australia.}

Received: 29 June 2016 Accepted: 30 June 2016

Published online: 3 August 2016

\section{References}

1. Singer P, Doig GS, Pichard C (2014) The truth about nutrition in the ICU. Intensive Care Med 40(2):252-255

2. Reigner J, Darmon M, Sommeville R, Borel AL, Garrouste-Orgeas M, Ruckly $S$ et al (2015) Impact of early nutrition and feeding route on outcomes of mechanically ventilated patients with shock: a post hoc marginal structural model study. Intensive Care Med 41(5):875-886

3. Leone M, Bechis C, Baumstarck K, Ouattara A, Collange O, Augustin P et al (2015) Outcome of acute mesenteric ischemia in the intensive care unit: a retrospective multicenter study of 780 cases. Intensive Care Med 41(4):667-676 (erratum Intensive Care Med 2015 May;41(5):966-8)

4. McClave SA, Taylor BE, Martindale RG, Warren MM, Johnson DR, Braunschwei $C$ et al (2016) Guidelines for the provision and assessment of nutrition support therapy in the adult critically ill patient: Society of Critical Care Medicine (SCCM) and American Society for Parenteral and Enteral Nutrition (ASPEN). JPEN J Parenter Enteral Nutr 40:159-211

5. Harvey SE, Parrott F, Harrison DA, Bear DE, Segargan E, Beale R et al (2014) Trial of the route of early nutritional support in critically ill adults. N Eng J Med 371:1673-1684

6. Elke G, van Zanten AR, Lemieux M, McCall M, Jeejeebhoy KN, Kott M, Jiang X, Day AG, Heyland DK (2016) Enteral versus parenteral nutrition in critically ill patients: an updated systematic review and meta-analysis of randomized controlled trials. Crit Care 20(1):117

7. Oshima T, Deutz NE, Doig G, Wischmeyer PE, Pichard C (2016) Proteinenergy nutrition in the ICU is the power couple: a hypothesis forming analysis. Clin Nutr 35(4):968-974 
8. Hickmann CE, Roeseler J, Castanares-Zapatero D, Herrera El, Mondogin A, Laterre PF (2015) Energy expenditure in critically ill physical therapy. Intensive Care Med 40:548-555

9. Singer P, Singer J (2016) Clinical guide for the use of metabolic carts: Indirect calorimetry-no longer the orphan of energy estimation. Nutr Clin Pract 31:30-38

10. Perez-Barcena J, Marse P, Zabalegui-Perez A, Corral E, Herran-Monge R, Gero-Escapa M et al (2014) A randomized trial of IV glutamine in trauma ICU patients. Intensive Care Med 40(4):539-547

11. Kagan I, Cohen J, Stein M, Bendavid I, Pinsker D, Silva V et al (2015) Preemptive enteral nutrition enriched with eicosapentaenoic acid, gamma-linolenic acid and antioxidants in severe multiple trauma: a prospective, randomized, double-blind study. Intensive Care Med 41(3):460-469

12. Zhu D, Zhang Y, Li S, Gan L, Feng H, Nie W (2014) Enteral omega-3 fatty acid supplementation in adult patients with acute respiratory distress syndrome: a systematic review of randomized controlled trials with metaanalysis and trial sequential analysis. Intensive Care Med 40(4):504-512
13. Kalfon P, Giraudeau B, Icahi C, Guerrini A, Brecht N, Cinotti R et al (2014) Tight computerized versus conventional glucose control in the ICU: a randomized controlled trial. Intensive Care Med 40(2):171-181

14. NICE-SUGAR Study Investigators for the Australian and New Zealand Intensive Care Society Clinical Trials Group and the Canadian Critical Care Trials Group, Finfer S, Chittock D, Li Y, Foster D, Dhingra V, Bellomo R, Cook D, Dodek P, Hebert P, Henderson W, Heyland D, Higgins A, McArthur C, Mitchell I, Myburgh J, Robinson B, Ronco J (2015) Intensive versus conventional glucose control in critically ill patients with traumatic brain injury: long-term follow-up of a subgroup of patients from the NICESUGAR study. Intensive Care Med 41 (6):1037-1047

15. Krag M, Perner A, Wettersley J, Wise MP, Hylander Moller M (2014) Stress ulcer prophylaxis versus placebo or no prophylaxis in critically ill patients. A systematic review of randomised clinical trials with meta-analysis and trial sequential analysis. Intensive Care Med 40(1):11-22 